\title{
Flexible Empirical Bayes Estimation For Wavelets
}

\author{
By Merlise Clyde and Edward I. George ${ }^{1}$
}

\section{SUMMARY}

Wavelet shrinkage estimation is an increasingly popular method for signal denoising and compression. Although Bayes estimators can provide excellent mean squared error (MSE) properties, selection of an effective prior is a difficult task. To address this problem, we propose Empirical Bayes (EB) prior selection methods for various error distributions including the normal and the heavier tailed Student $t$ distributions. Under such EB prior distributions, we obtain threshold shrinkage estimators based on model selection, and multiple shrinkage estimators based on model averaging. These EB estimators are seen to be computationally competitive with standard classical thresholding methods, and to be robust to outliers in both the data and wavelet domains. Simulated and real examples are used to illustrate the flexibility and improved MSE performance of these methods in a wide variety of settings.

Keywords: BAYESIAN MODEL AVERAGING; EM; HIERARCHICAL MODELS; MODEL SELECTION; MULTIPLE SHRINKAGE; OUTLIERS; ORTHOGONAL REGRESSION; ROBUSTNESS; THRESHOLDING.

\section{Introduction}

Wavelets are families of orthonormal basis functions that provide efficient representations for wide classes of functions, representations that are increasingly useful for signal denoising and compression. Towards this end and motivated by frequentist considerations, Donoho and Johnstone $(1994,1995)$ proposed a variety of wavelet shrinkage procedures for estimating a function observed with normal error. For this setup, recent papers such as Abramovich et al. (1998), Chipman et al. (1997) and Clyde et al. (1998) have proposed Bayesian shrinkage estimators that, for a variety of functions, yield improved performance over the earlier shrinkage methods. Although such improvements are available over broad prior classes, selection of the most effective prior can be especially difficult when little is known about the unknown function.

In this paper, we propose Empirical Bayes (EB) methods to resolve the prior selection problem for Bayesian estimation of wavelet coefficients. We develop these methods not only for the conventional nor-

\footnotetext{
${ }^{1}$ Merlise Clyde is Assistant Professor of Statistics, Institute of Statistics and Decision Sciences, Duke University, Durham, NC, 27708-0251, clyde@isds.duke.edu. Edward I. George hold the Ed and Molly Smith Chair and is Professor of Statistics, Department of MSIS, University of Texas, Austin, TX 78712-1175, egeorge@mail.utexas.edu. All correspondence should be addressed to Prof. Clyde. This work was supported by NSF grants DMS-96.26135, DMS-97.33013, and DMS-98.03756, and Texas ARP grants 003658.130 and 003658.452.
} 
mal error model, but also for heavier tailed error models such as Student $t$ distributions that can better accommodate extreme values and outliers. To handle these different error distributions, we begin with a hierarchical Bayesian wavelet model that uses a scale mixture of normals for the error distribution (section 2). We then propose EB methods that identify EB priors by using the data to estimate the hyperparameters and the error variance of this hierarchical model (section 3). Using these EB priors, we obtain threshold estimators based on model selection, and multiple shrinkage estimators based on model averaging. These EB procedures not only bypass the difficulty of specifying the hyperparameters in the prior distributions, but are also competitive with other shrinkage methods on computational grounds. These new EB estimators are seen to offer improved performance over previous methods in simulation studies (section 4), and are illustrated on real data (section 5).

\section{Statistical Model}

Suppose $Y=\left(Y_{1}, \ldots, Y_{n}\right)^{\prime}$ are $n$ equally spaced noisy observations of an unknown function $f=\left(f_{1}, f_{2}, \ldots, f_{n}\right)^{\prime}$ of interest, where $n$ is a power of 2 . Let $f=W \beta$ be an orthogonal wavelet decomposition of $f$, where $W$ is an $n \times n$ orthonormal matrix, and $\beta$ is the $n \times 1$ vector of wavelet coefficients for $f$. Let $D=W^{\prime} Y$ be the discrete wavelet transform (DWT) of $Y$, so that $D$ is the $n \times 1$ vector of empirical or observed wavelet coefficients. For this setup, we assume the model for the data in the wavelet domain is

$$
D_{j k}=\beta_{j k}+\epsilon_{j k}
$$

where $\epsilon_{j k}$ represents an additive error term. The doubly indexed subscripts reflect the multi-resolution nature of the wavelet decomposition; $j$ indexes scales or levels of resolution, and $k$ indexes locations.

A standard assumption is that the $\epsilon_{j k}$ are independent and normally distributed with mean zero and variance $\sigma^{2}$. While reasonable in many cases, extreme values and potential outliers may be more realistically modeled by heavy tailed error distributions. To extend the error distribution beyond the standard normal model, we replace it by a scale mixture of normals

$$
\begin{aligned}
\epsilon_{j k} \mid \lambda_{j k} & \sim N\left(0, \sigma^{2} / \lambda_{j k}\right) \\
\lambda_{j k} & \sim h
\end{aligned}
$$

where the $\epsilon_{j k}$ 's are conditionally independent, the $\lambda_{j k}$ 's are independent, and $h()$ is a scale mixing distribution on $(0, \infty)$. Scale mixtures of normals have been widely used in robustness studies and in outlier analysis (Andrews and Mallows 1974, West 1984, 1987, O’Hagan 1979, 1988) and include as special cases the normal, 
Laplace, exponential power, and Student $t$ distributions. The normal model is obtained when $\lambda_{j k} \equiv 1$, whereas independent errors with the $t_{\nu}$ distribution (the Student $t$ distribution with $\nu$ degrees of freedom) are obtained when $h$ is a Gamma distribution,

$$
\lambda_{j k} \sim \operatorname{Gamma}(\nu / 2,2 / \nu)
$$

with mean 1 . For notational convenience, we shall use $p_{h}(x ; 0, \sigma)$ to stand for the marginal density of $X$ when $X \mid \lambda \sim N\left(0, \sigma^{2} / \lambda\right)$ and $\lambda \sim h$.

For nondegenerate $h$, independent scale mixtures of normal errors in the wavelet domain corresponds to a scale mixture of normal errors in the data domain, though a different mixture. To see this, note that given $\Lambda$, the diagonal matrix with diagonal terms $\lambda_{j k}$, the data domain errors are normal with covariance matrix $\sigma^{2} W \Lambda^{-1} W^{\prime}$. Mixing over $\Lambda$ yields a different scale mixture with uncorrelated but typically dependent errors. (The unconditional covariance matrix, $\sigma^{2} W E_{h}\left(\Lambda^{-1}\right) W^{\prime}$, is proportional to the identity matrix as the $\lambda_{j k}$ are independent and identically distributed.) In particular, if the wavelet domain errors have independent $t_{\nu}$ distributions, then the data domain errors will be linear combinations of those $t_{\nu}$ random variables.

Although specification of independent $t_{\nu}$ distributions in the data domain may seem simpler than using other heavy tailed error distributions, exact calculations in the wavelet domain become intractable and require computationally intensive methods. Furthermore, if the only available information about the process is that the errors appear to be uncorrelated with a heavy tailed distribution (in either domain), then approximating the error distribution in the wavelet domain with independent $t_{\nu}$ distributions, perhaps with level dependent $\nu$, seems reasonable and is more appropriate than the standard normal model. At the very least, this model provides a useful approximation which, as we show, yields computationally tractable EB estimators for non-normal error distributions that are robust to outliers.

\subsection{Hierarchical Model}

The cornerstone of our Bayesian approach is an extension of the hierarchical normal mixture prior used by (Clyde et al. 1998). Based on the natural multilevel grouping of the wavelet coefficients, this distribution for the $\beta_{j k}$ 's at level $j$ is

$$
\begin{aligned}
\beta_{j k} \mid \lambda_{j k}^{*}, \gamma_{j k} & \sim N\left(0, \sigma^{2} c_{j} \gamma_{j k} / \lambda_{j k}^{*}\right) \\
\lambda_{j k}^{*} & \sim h^{*} \\
\gamma_{j k} & \sim \operatorname{Bernoulli}\left(\omega_{j}\right)
\end{aligned}
$$


where the $\beta_{j k}$ 's are conditionally independent, the $\lambda_{j k}^{*}$ 's and $\gamma_{j k}$ 's are independent, and $h^{*}()$ is a scale mixing distribution on $(0, \infty)$. We also assume that the $\beta_{j k}$ 's and the $\epsilon_{j k}$ 's are conditionally independent given the $\lambda_{j k}^{*}$ 's and the $\lambda_{j k}$ 's.

When the indicator variable $\gamma_{j k}=0$, the wavelet coefficient $\beta_{j k}=0$; when $\gamma_{j k}=1, \beta_{j k}$ is conditionally normal with a variance $\sigma^{2} c_{j} / \lambda_{j k}^{*}$. There are two fixed hyperparameters at each level: the expected fraction $\omega_{j}$ of non-zero wavelet coefficients at level $j$, and a fixed scaling factor $c_{j}>0$ for these non-zero coefficients. As with the error distribution, the introduction of random scale coefficients $\lambda_{j k}^{*}$ 's allows for a heavy-tailed distribution of the non-zero $\beta_{j k}$ 's. As will be seen in the next section, the effect of the choice of $h^{*}$ on posterior probabilities is strongly influenced by the choice of $h$ for the error distribution.

The final specification issue concerns the dependence between the $\lambda_{j k}^{*}$ 's and the $\lambda_{j k}$ 's. Because we do not expect a relationship between the signal and noise components, the most reasonable choice would be to specify these as independent, in which case the $\beta_{j k}$ 's and the $\epsilon_{j k}$ 's would be unconditionally independent. However, as will be seen, the EB calculations under this independent $\lambda$ specification become analytically intractable and must be carried out numerically.

To substantially ease the computational burden, it may be useful to consider the alternative specification obtained by forcing $\lambda_{j k}^{*}=\lambda_{j k}$ but maintaining the independence of the $\lambda_{j k}$ 's. Under this common $\lambda$ specification, each $\beta_{j k}$ and $\epsilon_{j k}$ pair are still uncorrelated (because of their conditional independence), but become unconditionally dependent through $\lambda_{j k}$. As is illustrated in Figure 1 in the next section, this dependence appears to have only a mild effect on posterior quantities of interest. Because the resulting posterior calculations are faster than the fully independent $\left(\lambda_{j k}, \lambda_{j k}^{*}\right)$ model and yield predictions that compare favorably with competing methods, this common $\lambda$ specification is at least a useful approximation to the full independence specification.

\subsection{Posterior Distributions}

The $\gamma_{j k}$ are a posteriori independent Bernoulli random variables, as a result of the conditional independence structure in the prior distributions and orthogonality of the DWT. The posterior probability that a coefficient is nonzero, $\pi\left(\gamma_{j k}=1 \mid Y\right)$, can be expressed as a function of the posterior odds, $O_{j k}$,

$$
\pi\left(\gamma_{j k}=1 \mid Y\right)=\frac{O_{j k}}{1+O_{j k}} \quad O_{j k}=\left(\frac{\omega_{j}}{1-\omega_{j}}\right) \frac{m_{1}\left(D_{j k} \mid \gamma_{j k}=1\right)}{m_{0}\left(D_{j k} \mid \gamma_{j k}=0\right)}
$$


where $m_{0}$ and $m_{1}$ are the marginal distributions of the data $D_{j k}$ when $\gamma_{j k}=0$ and 1 , respectively. The first of these is a standard normal scale mixture

$$
m_{0}\left(D_{j k} \mid \gamma_{j k}=0\right)=\int\left(\frac{\lambda_{j k}}{2 \pi \sigma^{2}}\right)^{1 / 2} \exp \left\{-\frac{1}{2} D_{j k}^{2} \lambda_{j k} / \sigma^{2}\right\} h\left(d \lambda_{j k}\right)=p_{h}\left(D_{j k} ; 0, \sigma\right),
$$

which can be computed in closed form in many cases of interest. The second of these is

$$
m_{1}\left(D_{j k} \mid \gamma_{j k}=1\right)=\iint \frac{1}{\sqrt{2 \pi \sigma^{2}}}\left(\frac{c_{j}}{\lambda_{j k}^{*}}+\frac{1}{\lambda_{j k}}\right)^{-1 / 2} \exp \left\{-\frac{1}{2} D_{j k}^{2}\left(\frac{\sigma^{2} c_{j}}{\lambda_{j k}^{*}}+\frac{\sigma^{2}}{\lambda_{j k}}\right)^{-1}\right\} h^{*}\left(d \lambda_{j k}^{*}\right) h\left(d \lambda_{j k}\right)
$$

when $\lambda_{j k}^{*}$ and $\lambda_{j k}$ are independent, and

$$
\begin{aligned}
m_{1}\left(D_{j k} \mid \gamma_{j k}=1\right) & =\int\left(\frac{\lambda_{j k}}{2 \pi \sigma^{2}\left(1+c_{j}\right)}\right)^{1 / 2} \exp \left\{-\frac{1}{2} D_{j k}^{2} \lambda_{j k} / \sigma^{2}\left(1+c_{j}\right)\right\} h\left(d \lambda_{j k}\right) \\
& =p_{h}\left(D_{j k} ; 0, \sigma \sqrt{\left(1+c_{j}\right)}\right)
\end{aligned}
$$

when $\lambda_{j k}^{*}=\lambda_{j k}$.

Table 1 summarizes several special cases to which we turn our attention. The Normal-Normal model for which the $\beta_{j k}$ 's and $\epsilon_{j k}$ 's have independent normal distributions is obtained when $\lambda_{j k}^{*} \equiv \lambda_{j k} \equiv 1$, and yields a closed form expression for the posterior odds $O_{j k}$ of $\gamma_{j k}=1$,

$$
O_{j k}=\left(1+c_{j}\right)^{-1 / 2}\left(\frac{\omega_{j}}{1-\omega_{j}}\right) \exp \left\{\frac{1}{2}\left(\frac{D_{j k}}{\sigma}\right)^{2}\left(\frac{c_{j}}{1+c_{j}}\right)\right\} .
$$

Next, to allow for heavy tailed error distributions, we consider several specifications under which the errors have the $t_{\nu}$ distribution corresponding to $\lambda_{j k}$ iid $\operatorname{Gamma}(\nu / 2,2 / \nu)$. The first of these is the Normal- $t$ model obtained by $\lambda_{j k}^{*} \equiv 1$, where the $\beta_{j k}$ 's are normal and independent of the $\epsilon_{j k}$ 's. Although this provides robustness to outliers thru the likelihood, the tails of the error distribution dominate the tails of the prior. This leads to posterior distributions that classify extremely large values of $\left|D_{j k}\right|$ as noise rather than signal, which may not be desirable. Posterior computations for this case must be done by numerical integration.

From a Bayesian prior robustness perspective, the tails of the prior should typically be at least as flat as the tails of the likelihood (Fan and Berger 1990). When the errors have a $t_{\nu}$ distribution, this is achieved when the $\beta_{j k}$ 's are iid $t_{\nu^{*}}$ with $\nu^{*} \leq \nu$, and are independent of the $\epsilon_{j k}$ 's. This corresponds to taking $\lambda_{j k}^{*}$ iid $\operatorname{Gamma}\left(\nu^{*} / 2,2 / \nu^{*}\right)$ with $\nu^{*} \leq \nu$, independently of the $\lambda_{j k}$ 's. We focus on the two special cases of this independent $t$ specification listed in Table 1: the $t$ - $t$ model with equal degrees of freedom, $\nu^{*}=\nu$, and the Cauchy- $t$ model with $\nu^{*}=1$. The marginal distribution in (10) can be obtained by first integrating out $\lambda_{j k}$ 


\begin{tabular}{|c|c|c|c|}
\hline Label & $\lambda_{j k}^{*}$ and $\lambda_{j k}$ Distributions & $\beta_{j k}$ and $\epsilon_{j k}$ Distributions & $\beta_{j k}$ and $\epsilon_{j k}$ Dependence \\
\hline \multirow{2}{*}{ Normal-Normal } & $\lambda_{j k}^{*}=\lambda_{j k} \equiv 1$ & Normal prior & Independent \\
& & Normal errors & \\
Normal- $t$ & $\lambda_{j k}^{*} \equiv 1$ & Normal prior & Independent \\
& $\lambda_{j k} \sim \operatorname{Gamma}(\nu / 2,2 / \nu)$ & $t_{\nu}$ errors & \\
$t-t$ & $\lambda_{j k}^{*} \sim \operatorname{Gamma}(\nu / 2,2 / \nu)$ & $t_{\nu}$ prior & Independent \\
& $\lambda_{j k} \sim \operatorname{Gamma}(\nu / 2,2 / \nu)$ & $t_{\nu}$ errors & \\
Cauchy- $t$ & $\lambda_{j k}^{*} \sim \operatorname{Gamma}(1 / 2,2)$ & Cauchy prior & Independent \\
& $\lambda_{j k} \sim \operatorname{Gamma}(\nu / 2,2 / \nu)$ & $t_{\nu}$ errors & \\
Bivariate $t$ & $\lambda_{j k}^{*}=\lambda_{j k}$ & $t_{\nu}$ prior & Uncorrelated \\
& $\lambda_{j k} \sim \operatorname{Gamma}(\nu / 2,2 / \nu)$ & $t_{\nu}$ errors & But Dependent \\
\hline
\end{tabular}

Table 1: Joint distributions of $\beta_{j k}$ and $\lambda_{j k}$

and $\lambda_{j k}^{*}$

$$
m_{1}\left(D_{j k} \mid \gamma_{j k}=1\right)=\int \frac{K_{\nu}}{\sigma\left(1+\frac{\left(D_{j k}-\beta_{j k}\right)^{2}}{\nu \sigma^{2}}\right)^{(\nu+1) / 2}} \frac{K_{\nu^{*}}}{\sigma \sqrt{c_{j}}\left(1+\frac{\beta_{j k}^{2}}{\nu^{*} c_{j} \sigma^{2}}\right)^{\left(\nu^{*}+1\right) / 2}} d \beta_{j k}
$$

reducing (10) to a one dimensional integral over $\beta_{j k}$ of the product of the two $t$ densities $\left(K_{\nu}\right.$ and $K_{\nu^{*}}$ are the normalizing constants of the $t_{\nu}$ and $t_{\nu^{*}}$ densities, respectively). Furthermore, for cases where $\nu$ and $\nu^{*}$ are both odd integers, Fan and Berger (1990) have shown that exact closed form expressions for (13) and the posterior mean of $\beta_{j k}$ can be obtained. Although the marginal distribution in such cases can be expressed in closed form (the lengthy expressions are omitted), it is a complicated function of $c_{j}$ and $\sigma^{2}$. As a result, numerical methods are required to obtain the corresponding EB estimates discussed in Section 3.

Finally, a prior setup for $t_{\nu}$ distribution errors, which lessens the above computational burdens, is the Bivariate $t$ model where the $\left(\beta_{j k}, \epsilon_{j k}\right)$ pairs have independent bivariate $t_{\nu}$ distributions corresponding to $\lambda_{j k}^{*}=\lambda_{j k} \sim \operatorname{Gamma}(\nu / 2,2 / \nu)$. The marginal distributions $m_{0}$ and $m_{1}$ are both rescaled $t_{\nu}$ distributions, and the odds expression simplifies to

$$
O_{j k}=\left(1+c_{j}\right)^{-1 / 2}\left(\frac{\omega_{j}}{1-\omega_{j}}\right)\left[\frac{1+\frac{1}{\nu}\left(\frac{D_{j k}^{2}}{\sigma^{2}\left(1+c_{j}\right)}\right)}{1+\frac{1}{\nu}\left(\frac{D_{j k}^{2}}{\sigma^{2}}\right)}\right]^{-(\nu+1) / 2} .
$$

Although $\beta_{j k}$ and $\epsilon_{j k}$ are dependent here, they remain uncorrelated. As will be seen below, posterior probabilities under the Bivariate $t$ closely approximate posterior probabilities under the $t$ - $t$, but can be computed in less than $1 / 7$ th the time. 
Figure 1 shows the posterior probability $\pi\left(\gamma_{j k}=1 \mid Y\right)$ as a function of $\left|t_{j k}\right|=\left|D_{j k}\right| / \sigma$ for various choices of $c_{j}$ under each of the priors listed in Table 1 for $\nu=5$. (Numerical integration was required to compute the curves for the Normal- $t$ model). Under the Normal-Normal model when $c_{j}>0, \pi\left(\gamma_{j k}=1 \mid Y\right)$ increases rapidly in $\left|t_{j k}\right|$ to 1 , thus treating extreme values as signal. In sharp contrast, under the Normal- $t$ model, $\pi\left(\gamma_{j k}=1 \mid Y\right)$ eventually decreases in $\left|t_{j k}\right|$, causing it to treat extreme values as noise. This occurs because the tails of the $t_{\nu}$ distribution eventually dominate the normal prior. This behavior, which is more evident when $c_{j}$ is small, is undesirable for functions that have a few very large coefficients at the finest level because the Normal- $t$ model would tend to treat them as outliers.

The Cauchy- $t$ curve corresponds to an independent $t$ specification with a Cauchy prior $\left(\nu^{*}=1\right)$ and a $t_{5}$ error distribution. The Cauchy prior is the easiest of the independent $t$ priors to handle computationally, and has desirable robustness properties. Like the Normal-Normal, $\pi\left(\gamma_{j k}=1 \mid Y\right)$ under the Cauchy- $t$ eventually approaches 1 for large $\left|D_{j k}\right|$. However, it does so more slowly, exhibiting greater uncertainty that an observation represents signal.

The $\pi\left(\gamma_{j k}=1 \mid Y\right)$ curves for both the Bivariate $t$ and $t$ - $t$ model (with $\nu^{*}=\nu=5$ ) are very similar, especially for $c_{j}$ large. Providing a compromise between the Normal-Normal and the Normal- $t$ models, both add robustness while avoiding the Normal- $t$ non-monotonicity phenomenon. For the Bivariate $t$ model, the posterior odds $O_{j k}$ has a limit of $\left(\omega_{j} /\left(1+\omega_{j}\right)\right)\left(1+c_{j}\right)^{\nu_{j} / 2}$, as $\left|t_{j k}\right|$ goes to infinity. For large $c_{j}$, this behaves effectively like the normal model. However, compared to both the Normal-Normal and the Cauchy- $t$ models, for small $c_{j}$ (where it is difficult to distinguish between signal and noise), there is more uncertainty about whether a coefficient is nonzero, which does not disappear for large $\left|D_{j k}\right|$. Because of the availability of simple closed form posterior expressions, the Bivariate $t$ model can be seen as an attractive approximation to the $t$ - $t$ model, providing a reasonable compromise between robust estimation and computational ease. Because of the similarity of the Bivariate $t$ and the $t$ - $t$, we will proceed to focus our attention on the Normal-Normal, Bivariate $t$ and the Cauchy- $t$ models.

\section{Empirical Bayes Methods}

Implementation of the Bayes hierarchical model (5)-(7), with fixed hyperparameter values requires the specification of $c_{j}$ and $\omega_{j}$ at each level $j$ of the wavelet decomposition. Unfortunately, meaningful pre-specification of these is difficult at best. Although one's prior beliefs might reflect an ordering of the $c_{j}$ and $\omega_{j}$ by level, it is difficult to subjectively elicit additional information. A fully automatic Empirical Bayes (EB) approach that estimates $c_{j}, \omega_{j}$ and $\sigma^{2}$ from the data is appealing because it avoids this difficulty and the possibility 

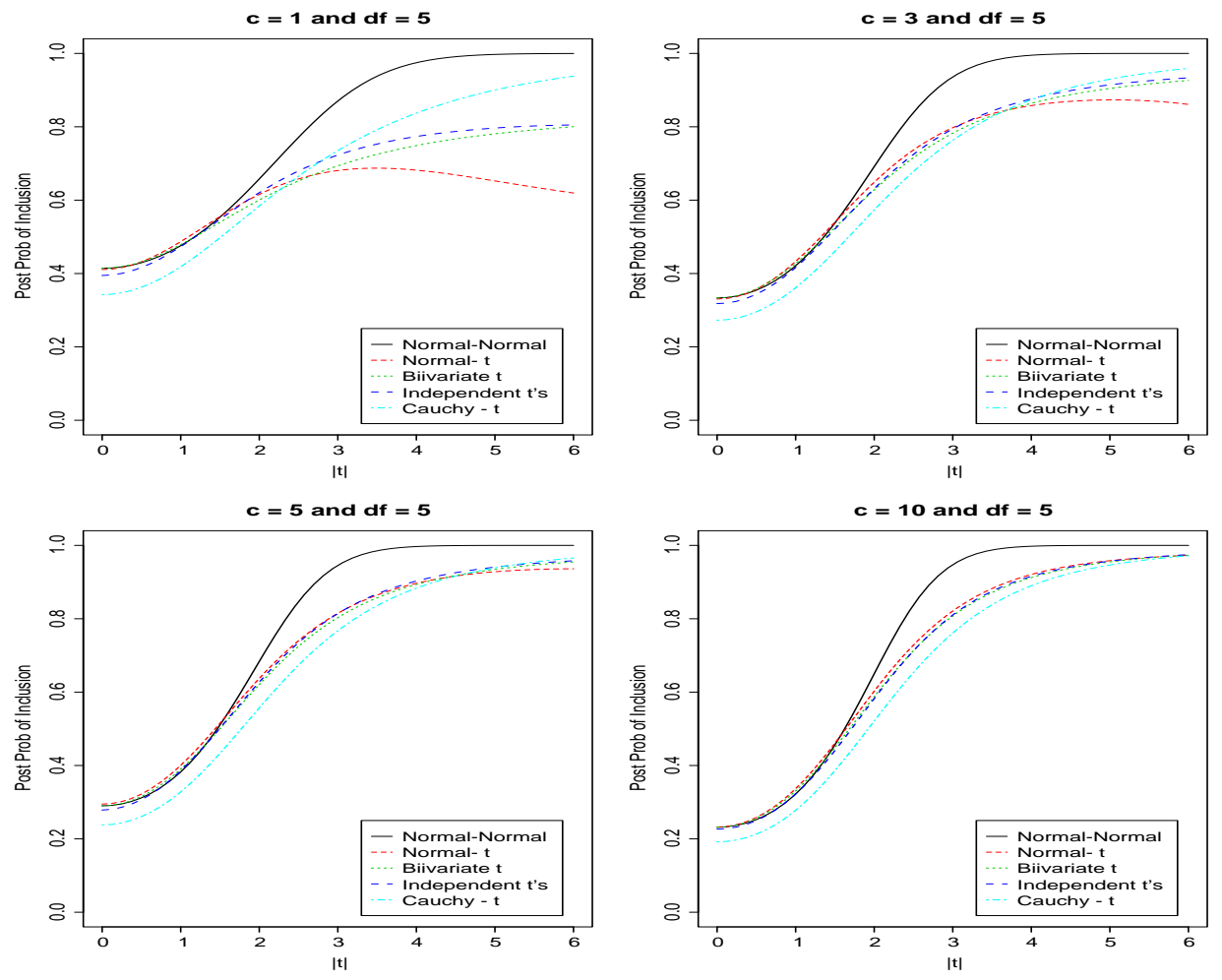

Figure 1: The posterior probability of inclusion, $\pi\left(\gamma_{j k}=1 \mid Y\right)$, as a function of $\left|t_{j k}\right|=\left|D_{j k}\right| / \sigma$ under the specifications listed in Table 1 . In all cases $\omega=.5$

of specifying values which are in conflict with the data.

The EB approach we propose is based on using maximum marginal likelihood (MML) estimates of the hyperparameters, and extends the approach of George and Foster (1997) for the normal linear model. Because closed form EB estimates of $c_{j}$ and $\omega_{j}$ are generally unavailable, numerical methods must be used. For this purpose, we consider several computationally attractive alternatives: direct numerical maximization, EM algorithms and a conditional maximum likelihood (CML) approximation.

\subsection{Direct Maximum Likelihood Estimation}

Marginally, the observations $D_{j k}$ given $c_{j}, \omega_{j}$, and $\sigma^{2}$, are independently distributed as a mixture of $m_{0}$ as in (9) and $m_{1}$ as given by (11) or (13), with respective weights $\omega_{j}$ and $1-\omega_{j}$. The log likelihood $\mathcal{L}$ for $c=\left(c_{1}, \ldots c_{J}\right)^{\prime}, \omega=\left(\omega_{1}, \ldots, \omega_{J}\right)^{\prime}$ and $\sigma^{2}$ is then

$$
\mathcal{L}\left(c, \omega, \sigma^{2}\right)=\sum_{j}\left\{\sum_{k} \log \left[\omega_{j} m_{1}\left(D_{j k} ; c_{j}, \sigma\right)+\left(1-\omega_{j}\right) m_{0}\left(D_{j k} ; \sigma\right)\right]\right\} .
$$

Although closed form solutions for the marginal maximum likelihood (MML) estimates $\hat{c}_{j}$ and $\hat{\omega}_{j}$ are generally unavailable, standard iterative methods can be used for direct maximization of the log likelihood (15). In 
particular, we have found nonlinear Gauss-Seidel iteration (Thisted 1988, pp 187-188) to work well. This entails iterating between finding the maximizing $\hat{c}_{j}$ given $\left(\hat{\omega}_{j}, \hat{\sigma}^{2}\right)$, the maximizing $\hat{\omega}_{j}$ given $\left(\hat{c}_{j}, \hat{\sigma}^{2}\right)$ for each $j$, and the maximizing $\hat{\sigma}^{2}$ given $\left(\hat{c}_{1}, \hat{\omega}_{1}\right), \ldots,\left(\hat{c}_{J}, \hat{\omega}_{J}\right)$. As the Hessian of the log likelihood is in general not positive definite for all values of $c_{j}, \omega_{j}$, and $\sigma^{2}$, convergence may only be to a local mode. However, we have achieved reasonable success using the MAD estimate $\hat{\sigma}=\operatorname{Median}\left(\left|D_{1 k}\right|\right) / 0.6745$ (Donoho et al. 1995) as an initial value for $\sigma$, and $\hat{\omega}_{j}$ equal to the number of observed wavelet coefficients that exceed $\sqrt{2 \log n} \hat{\sigma}$ based on hard thresholding.

\subsection{Maximum Likelihood Estimation using the EM Algorithm}

For the Normal-Normal model with a fixed value for $\sigma^{2}$, Johnstone and Silverman (1998) have recently proposed an EM algorithm based on a metric inequality. To implement a more general EM algorithm here, we consider the augmented $\log$ likelihood given $D$ and latent or "missing" variables $\lambda$ and $\gamma$. This leads to an alternative method for maximizing the marginal likelihood in (15). Unless, otherwise noted, we shall now restrict attention to common $\lambda$ models such as the Normal-Normal and the Bivariate $t$ where $\lambda_{j k}^{*}=\lambda_{j k}$, which leads to closed form iterative solutions for the parameters.

Let $\tau_{j}^{-1}=\left(1+c_{j}\right) \sigma^{2}$, where $\tau_{j}$ corresponds to the unknown precision parameter for the data, $D_{j k}$, given $\gamma_{j k}=1$ and let $\phi=1 / \sigma^{2}$, the precision when $\gamma_{j k}=0$. The log likelihood for $\tau=\left(\tau_{1}, \ldots, \tau_{J}\right)^{\prime}, \omega$ and $\phi$ based on the "augmented" or "completed" data, $X=(D, \lambda, \gamma)$, is

$$
\begin{aligned}
\mathcal{L}(\omega, \tau, \phi \mid D, \lambda, \gamma)= & \sum_{j}\left[n_{j} \log \left(1-\omega_{j}\right)+\log \left(\frac{\omega_{j}}{1-\omega_{j}}\right) \sum_{k} \gamma_{j k}\right] \\
& +\frac{1}{2} \sum_{j}\left[\log \left(\tau_{j}\right) \sum_{k} \gamma_{j k}-\tau_{j} \sum_{k} D_{j k}^{2} \lambda_{j k} \gamma_{j k}\right] \\
& +\frac{1}{2} \log (\phi) \sum_{j k}\left(1-\gamma_{j k}\right)-\frac{1}{2} \phi \sum_{j, k} D_{j k}^{2} \lambda_{j k}\left(1-\gamma_{j k}\right) \\
& +\sum_{j k} \log \left(h\left(\lambda_{j k}\right)\right)
\end{aligned}
$$

and belongs to a regular exponential family of the form $a(\theta)^{\prime} b(X)+c(\theta)+d(X)$ where $\theta=(\omega, \tau, \phi), a(\theta)$ is the $2 J+1$ dimensional vector of natural parameters, and $b(X)$ is the $2 J+1$ vector of sufficient statistics with components $\left(\sum_{k} \gamma_{j k}, \sum_{k} D_{j k}^{2} \lambda_{j k} \gamma_{j k}\right)$ for $j=1, \ldots J$ and $\sum_{j k} D_{j k}^{2} \lambda_{j k}\left(1-\gamma_{j k}\right)$.

The E-step of the EM algorithm consists of computing the expectations of the sufficient statistics with respect to the distribution of $(\lambda, \gamma)$ given $D, c$, and $\omega$. The posterior mean of $\gamma_{j k}$ is

$$
E\left(\gamma_{j k} \mid D\right)=\hat{\gamma}_{j k}^{(i)}=\frac{O_{j k}^{(i)}}{1+O_{j k}^{(i)}}
$$


where $O_{j k}^{(i)}$ is the posterior odds (8) evaluated using the current estimates $\hat{c}_{j}^{(i)}$ and $\hat{\omega}_{j}^{(i)}$. For the Bivariate $t$ model, where $\lambda_{j k} \sim \operatorname{Gamma}\left(\nu_{j} / 2,2 / \nu_{j}\right)$, the posterior distribution of $\lambda_{j k}$ given $\gamma_{j k}$ is Gamma, with

$$
\begin{aligned}
E\left(\lambda_{j k} \gamma_{j k} \mid D\right) & =\frac{\nu_{j}+1}{\nu_{j}+D_{j k}^{2} \hat{\gamma}_{j}^{(i)}} \hat{\gamma}_{j k}^{(i)}=\left[\hat{\lambda}_{j k} \hat{\gamma}_{j k}\right]^{(i)} \\
E\left(\lambda_{j k}\left(1-\gamma_{j k}\right) \mid D\right) & =\frac{\nu_{j}+1}{\nu_{j}+D_{j k}^{2} \hat{\phi}^{(i)}}\left(1-\hat{\gamma}_{j k}^{(i)}\right)=\left[\hat{\lambda}_{j k}\left(1-\hat{\gamma}_{j k}\right)\right]^{(i)} .
\end{aligned}
$$

The M-step consists of maximizing the augmented likelihood with the latent data now replaced by their posterior expected values, resulting in

$$
\begin{aligned}
\hat{\sigma}^{2^{(i+1)}} & =\frac{\sum_{j k} D_{j k}^{2}\left[\hat{\lambda}_{j k}\left(1-\hat{\gamma}_{j k}\right)\right]^{(i)}}{n-\sum_{j k} \hat{\gamma}_{j k}^{(i)}} \\
\hat{\omega}_{j}^{(i+1)} & =\frac{\sum_{k} \hat{\gamma}_{j k}^{(i)}}{n_{j}} \\
\hat{c}_{j}^{(i+1)} & =\max \left(0, \frac{\sum_{k} D_{j k}^{2}\left[\hat{\lambda}_{j k} \hat{\gamma}_{j k}\right]^{(i)}}{\hat{\sigma}^{2^{(i+1)}} \sum_{k} \hat{\gamma}_{j k}^{(i)}}-1\right) .
\end{aligned}
$$

The EM algorithm for the Normal-Normal model is obtained by simply setting $\hat{\lambda}_{j k} \equiv 1$ in the above.

If the parameter estimates are in the interior of the parameter space, the solutions above are the unique solutions (conditional on the values of latent data) because the complete data belong to a regular exponential family. The $\mathrm{E}$ and $\mathrm{M}$ steps are repeated until the estimates converge, and yield a stationary point of the marginal likelihood (15). However, as in the case of direct maximization of the marginal likelihood using Gauss-Seidel or other methods, the EM algorithm may converge to a local mode. Because the convergence rate of the standard EM algorithm is linear (Dempster, Laird, and Rubin 1977), the direct maximization methods described above may result in faster convergence. However, the iterative solutions are available in closed form for the common scale $\lambda_{j k}$ model and provide some insight into the problem and connections to the CML estimates of George and Foster (1997). The M-step estimates of $\sigma^{2}$ and $w_{j}$ have natural interpretations; the estimate of $\sigma^{2}$ is the ratio of the posterior expected error sum of squares to the posterior expected df, and the estimate of $w_{j}$ is the posterior expected fraction of nonzero coefficients.

While it is not necessary to introduce $\lambda_{j k}$ as a latent variable, this does permit closed form iterative expressions for the hyperparameter estimates of the Bivariate $t$ model. For models where the complete data are not from a regular exponential family, such as in the Cauchy- $t$ model, numerical optimization is required to carry out the M-step for $c_{j}$ and $\sigma^{2}$, although $\hat{\omega}_{j}$ has the same form as in (18). In practice, we have noticed very little difference between MLEs using the EM algorithm or the direct maximization approach for the Normal-Normal, Bivariate $t$ or Cauchy- $t$ models. 


\subsection{Conditional Likelihood Approximations}

For the Normal-Normal model, the conditional maximum likelihood (CML) approach of George and Foster (1997) provides a fast alternative to direct maximization and EM, and can be viewed as taking the complete data likelihood (16) and evaluating it at the mode for $\gamma$, rather than using the posterior mean, as in the EM algorithm. Let $q_{j}=\sum_{k} \gamma_{j k}$ denote the the number of nonzero wavelet coefficients at level $j$. For fixed $j$, let $D_{j(k)}^{2}$ denote the sorted values (in decreasing order) of $\left(D_{j k} / \hat{\sigma}\right)^{2}$, where $\hat{\sigma}^{2}$ corresponds to the MAD estimate of $\sigma^{2}$. The most likely model with $q_{j}$ nonzero components at level $j, \tilde{\gamma}\left(q_{j}\right)$ has elements $\tilde{\gamma}_{j(k)}=1$ if $k \leq q_{j}$, and 0 otherwise.

For each value of $q_{j}$, the values of $c_{j}$ and $\omega_{j}$ that maximize the conditional log likelihood are

$$
\omega_{j}\left(q_{j}\right)=\frac{\sum_{k} \tilde{\gamma}_{j(k)}\left(q_{j}\right)}{n_{j}}=q_{j} / n_{j} \quad c_{j}\left(q_{j}\right)=\max \left\{0, \frac{\sum_{k} D_{j(k)}^{2} \tilde{\gamma}_{j(k)}\left(q_{j}\right)}{\sum_{k} \tilde{\gamma}_{j(k)}\left(q_{j}\right) \hat{\sigma}^{2}}-1\right\} .
$$

The CML estimators have the same form as the EM MML estimators, and are the same when the posterior distribution for $\gamma_{j k}$ is degenerate at 1 or 0 , where we have perfect classification of the observation into noise or signal. For large values of $c_{j}$, there is often very good separation of signal and noise, resulting in little bias of the CML estimators.

The difference between the MML and the CML estimates will be the most extreme when the posterior mean of $\gamma_{j k}$ is 0.5 and when $c_{j}$ is small. The simulation studies described later suggest, however, that the EB estimators using the conditional estimates have better MSE performance than earlier shrinkage estimators. Although this approach does not readily extend to the case of $t$ errors without further approximations, the Normal-Normal model solutions can be used as starting values for iterative methods such as the Gauss-Seidel or EM algorithms.

\subsection{Empirical Bayes Estimators}

We consider two types of Empirical Bayes estimators: threshold shrinkage estimators based on model selection, and multiple shrinkage estimators based on model averaging. The EB estimators are obtained as posterior means, treating the MML hyperparameter estimates as if they were fixed in advance. The $\beta_{j k} \mathrm{~s}$ under the common $\lambda$ model are a posteriori conditionally independent,

$$
p\left(\beta_{j k} \mid \gamma_{j k}, \lambda_{j k}, Y\right) \sim N\left(\gamma_{j k} \frac{c_{j}}{1+c_{j}} D_{j k}, \frac{\gamma_{j k}}{\lambda_{j k}} \sigma^{2} \frac{c_{j}}{1+c_{j}}\right) .
$$

Expressions for the posterior mean of $\beta_{j k}$ given $\gamma_{j k}=1$ under the Cauchy- $t$ model can be obtained from equations in Fan and Berger (1990). Unless noted otherwise, all results below hold for the Cauchy- $t$ model, with $\left(c_{j} /\left(1+c_{j}\right)\right) D_{j k}$ replaced by $E\left[\beta_{j k} \mid \gamma_{j k}=1, Y\right]$ computed under the Cauchy- $t$ model. 


\section{Threshold Shrinkage Estimators}

Thresholded shrinkage estimators are obtained as posterior means conditionally on the highest posterior probability model or $\gamma=\left(\gamma_{11}, \ldots, \gamma_{J K}\right)$. (As in the variable selection context, a "model" can be identified with $\gamma$ ). As a consequence of the conditional independence structure, the posterior probability of $\gamma$ is of the form

$$
p(\gamma \mid D)=\prod_{j k} \pi\left(\gamma_{j k}=1 \mid D_{j k}\right)^{\gamma_{j k}}\left(1-\pi\left(\gamma_{j k}=1 \mid D_{j k}\right)\right)^{\left(1-\gamma_{j k}\right)},
$$

a product of independent Bernoulli probabilities. Because of the product structure, the highest posterior probability model, $\hat{\gamma}=\left(\hat{\gamma}_{11}, \ldots, \hat{\gamma}_{J K}\right)$, is obtained by setting $\hat{\gamma}_{j k}=1$ if $\pi\left(\gamma_{j k}=1 \mid D_{j k}\right) \geq 0.5$ and $\hat{\gamma}_{j k}=0$ otherwise. The posterior mean under $\hat{\gamma}$ for the common $\lambda$ model is then

$$
E\left(\beta_{j k} \mid \hat{\gamma}, Y\right)=\hat{\gamma}_{j k} \frac{\hat{c}_{j}}{1+\hat{c}_{j}} D_{j k}
$$

This threshold shrinkage estimator thresholds the data by setting $\hat{\beta}_{j k}=0$ whenever $\hat{\gamma}_{j k}=0$, and then shrinks the remaining coefficients. This is useful for compression problems where dimension reduction and elimination of negligible coefficients is important. Because the inverse DWT (IDWT) is linear, the posterior mean of $f$ under model $\hat{\gamma}$ is obtained by applying the IDWT to the posterior mean of $\beta$ under model $\hat{\gamma}$.

\section{Multiple Shrinkage Estimators}

Instead of conditioning on a single model as above, an estimator of $\beta_{j k}$ which incorporates uncertainty about $\gamma_{j k}$, is the unconditional posterior mean

$$
E\left(\beta_{j k} \mid Y\right)=\pi\left(\gamma_{j k}=1 \mid Y\right) \frac{\hat{c}_{j}}{1+\hat{c}_{j}} D_{j k}
$$

Note that $\pi\left(\gamma_{j k}=1 \mid Y\right)=\pi\left(\gamma_{j k}=1 \mid D_{j k}\right)$ is given by (8), where the marginals $m_{0}$ and $m_{1}$ are based on the EB hyperparameter estimates $\hat{c}_{j}$ and $\hat{\omega}_{j}$. For specifications such as the Normal-Normal and the Bivariate $t$, the resulting closed form expressions for $\pi\left(\gamma_{j k}=1 \mid Y\right)$ and the EB multiple shrinkage estimators can be computed quickly once we have the hyperparameter estimates. In contrast, the multiple shrinkage estimator for the Cauchy- $t$ takes approximately 30 times longer (not including the extra time necessary to obtain the hyperparameter estimates) for $n=1024$. The posterior mean of $f$ is obtained by applying the IDWT to the multiple shrinkage estimator of $\beta$.

The estimator (24) is a multiple shrinkage estimator (George 1986, Clyde et al. 1998) and corresponds to model averaging. Because of the form of the conditional distribution (21), the usual posterior weighted sum 
of conditional expectations reduces here to the simple form $(24)$ that inserts $\pi\left(\gamma_{j k}=1 \mid Y\right)$ as an additional shrinkage factor. As opposed to the threshold shrinkage estimator, which sets negligible coefficients to zero, the multiple shrinkage estimator provides nonzero estimates for all coefficients for which $\hat{\pi}\left(\gamma_{j k}=1 \mid D_{j k}\right) \neq 0$.

For problems where threshold shrinkage rather than multiple shrinkage is desired, it is worth noting that the threshold estimator (23) minimizes squared error loss for estimation or prediction under model selection (J.O. Berger, personal communication). We provide a proof for the case of wavelet regression. Let $\hat{f}_{\gamma}$ denote the posterior mean for $f$ under model $\gamma$ corresponding to $(23)$, and let $\hat{f}$ denote the unconditional posterior mean corresponding to $(24) ; \hat{f}$ is the Bayes rule under squared error loss for estimation of $f$. The best single model $\gamma^{*}$ for estimating $f$ under posterior expected squared error loss is the model such that $\hat{f}_{\gamma}$ is closest to $\hat{f}$, or that minimizes

$$
\left(\hat{f}_{\gamma}-\hat{f}\right)^{\prime}\left(\hat{f}_{\gamma}-\hat{f}\right)
$$

Because $W$ is orthonormal, this can be equivalently expressed in the wavelet domain by

$$
\left(\hat{f}_{\gamma}-\hat{f}\right)^{\prime} W W^{\prime}\left(\hat{f}_{\gamma}-\hat{f}\right)=\sum_{j k}\left(\gamma_{j k}-\pi\left(\gamma_{j k}=1 \mid Y\right)\right)^{2}\left(E\left[\beta_{j k} \mid Y, \gamma_{j k}=1\right]\right)^{2}
$$

The last expression is minimized by the model with $\gamma_{j k}^{*}=1$ if $\pi\left(\gamma_{j k}=1 \mid Y\right) \geq 0.5$ and with $\gamma_{j k}^{*}=0$ if $\pi\left(\gamma_{j k}=1 \mid Y\right)<0.5$, which is equivalent to the highest posterior model when the posterior distribution for $\gamma$ has the form given by (22) and is the median probability model as defined by Berger.

Although the multiple shrinkage estimator tends to outperform the threshold shrinkage estimator in terms of squared error loss, sometimes the improvements are small. Such gains may not compensate for the costs of including additional terms, as might occur, for example, in compression problems. We will see in the next section that both (23) and (24) appear to offer improved frequentist risk performance over several classical estimators.

\section{Simulations}

To investigate the practical potential of our EB method, we evaluated the performance of the EB estimators under the Normal-Normal, the Bivariate $t$ and Cauchy- $t$ specifications, using noisy versions of the four test functions "blocks", "bumps", "doppler", "heavisine" proposed by Donoho and Johnstone (1994). In every case, the functions were scaled so that the signal-to-noise ratio was 7 , (the ratio of the standard deviation of the function values $f_{i}$ to the standard deviation of the noise). We compared this performance with that of several existing shrinkage strategies: HARD - Hard thresholding with the universal rule, SOFT - Soft thresholding with the universal rule, and SURE - SureShrink adaptive shrinkage rule as implemented in 
blocks

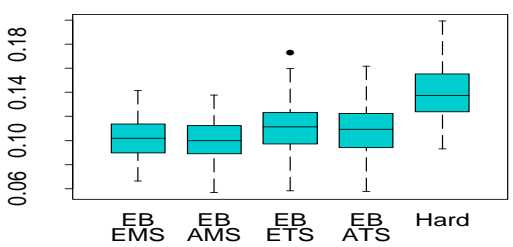

doppler

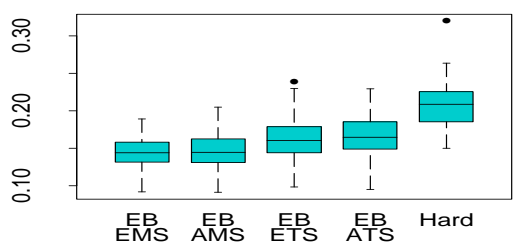

bumps

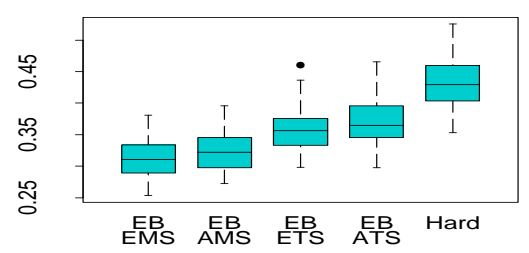

heavisine

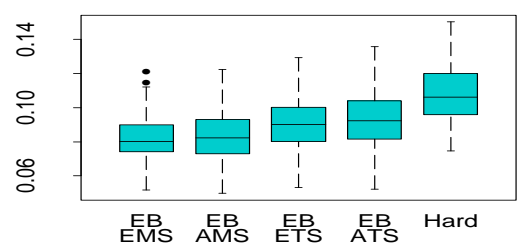

Figure 2: MSE for the EB threshold shrinkage (TS) and multiple shrinkage (MS) estimators using exact (E) and approximate (A) hyperparameter estimates, and the HARD estimator.

S+Wavelets, Donoho and Johnstone $(1994,1995)$. The wavelet bases were chosen to match those used by Donoho and Johnstone (1995). To assess robustness, we considered both normal and heavy-tailed distributions for the noise. Performance was evaluated by the average mean squared error (MSE) over 100 simulations for each problem,

$$
\mathrm{MSE}=\frac{1}{100} \sum_{l=1}^{100} \sum_{i=1}^{n} \frac{\left(f_{i}-\hat{f}_{i}^{l}\right)^{2}}{n},
$$

where $f_{i}$ is the true signal and $\hat{f}_{i}^{l}$ is the estimate of the function from simulation $l$.

\subsection{Normal Errors}

For our first set of simulations, we repeatedly added $n=1024$ independent $N(0,1)$ errors to each of the four test functions. For the Normal-Normal specification, we first compared the threshold shrinkage estimator, TS in (23) and the multiple shrinkage estimator, MS in (24) to HARD thresholding. We also compared the threshold shrinkage and multiple shrinkage estimators using EB hyperparameter estimates based on the exact (E) marginal likelihood (MML) and on the approximate (A) conditional likelihood (CML) discussed in Section 3.

Figure 2 presents boxplots of MSEs of these five estimators, and shows that all four EB estimators are superior to HARD in this setting. Of the EB estimators, MS is superior to TS for both exact and approximate hyperparameter estimates. However, estimates based on the exact marginal likelihood are at most slightly better than those based on the rapidly computable approximate conditional likelihood. 
blocks

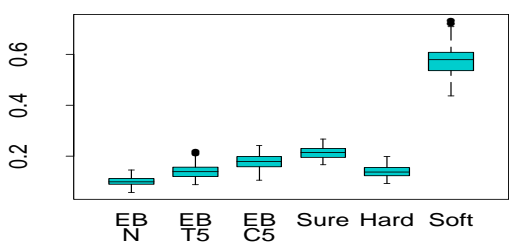

doppler

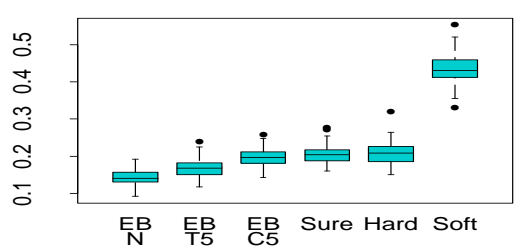

bumps
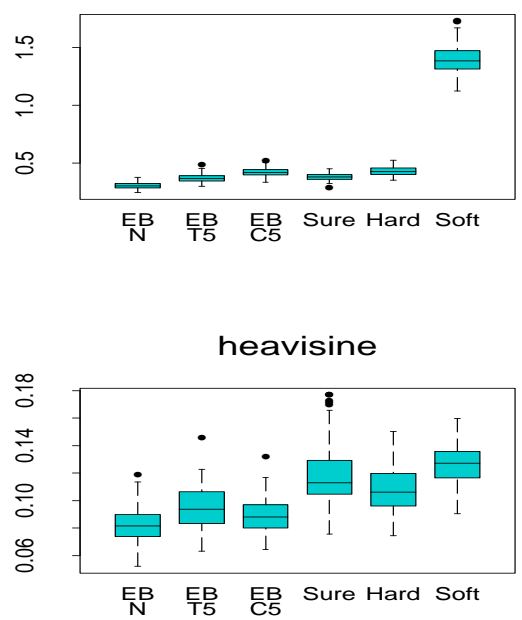

Figure 3: MSE comparisons for data simulated with normal errors. The EB estimators are all based on multiple shrinkage.

Restricting attention now to multiple shrinkage estimators using exact marginal likelihood hyperparameter estimates, Figure 3 compares the EB estimators for the Normal-Normal, the Bivariate $t_{5}$ (with 5 df) and the Cauchy- $t_{5}$ specifications, labeled EB N, EB T5, and EB C5, to the SURE, HARD, and SOFT estimators. The EB N estimator exhibits excellent MSE performance compared to the other methods. However, EB T5 performs nearly as well, demonstrating only a small loss of efficiency and is equal or better than HARD, SURE or SOFT.

Figure 4 shows the distribution of the MLEs of $\sqrt{c_{j}}$ and $\omega_{j}$ under the normal model for the four test functions. Variation of the estimates across levels is striking, revealing strong decay in both $\omega_{j}$ and $c_{j}$ but different rates across functions. Although such decay might be roughly anticipated using a fixed hyperparameter Bayes setup with subjective prior inputs, it is difficult to pre-specify the appropriate magnitude and rate of decay. MSE performance comparisons with the fixed hyperparameter Bayes estimators of Clyde et al. (1998) again favored our EB estimators.

\subsection{Student $t$ Errors in the Wavelet Domain}

For our second set of simulations, we repeatedly added $n=1024$ independent $t_{5}$ errors to the true wavelet coefficients for each of the four test functions. Figure 5 compares MSEs for the multiple shrinkage EB estimators EB N, EB T5, and EB C5, with those for the SURE, HARD, and SOFT estimators.

Comparing Figure 5 with Figure 3 reveals that the MSE performance of all the estimators tends to 

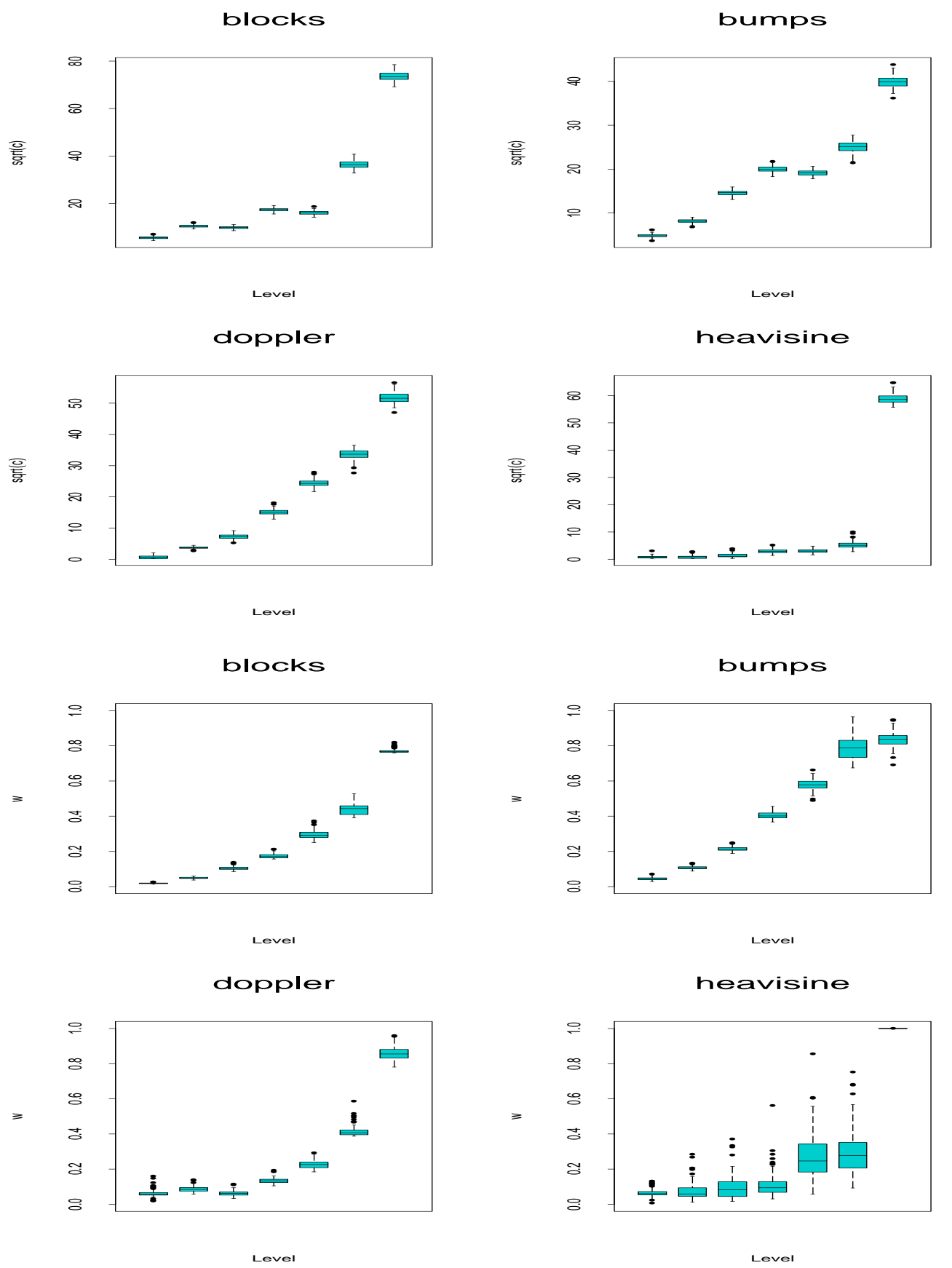

Figure 4: EB estimators of $\sqrt{c_{j}}$ and $\omega_{j}$ for data simulated with normal errors. 
blocks

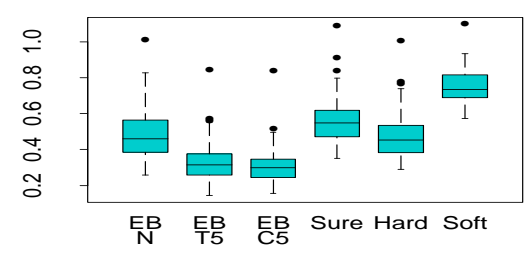

doppler

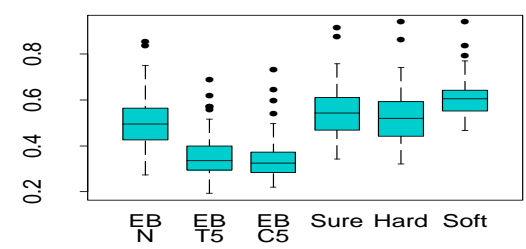

bumps

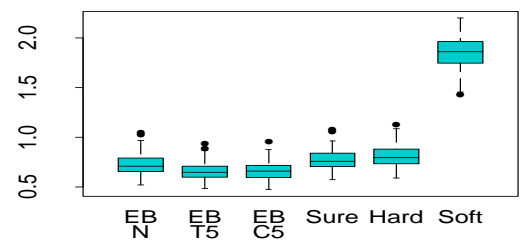

heavisine

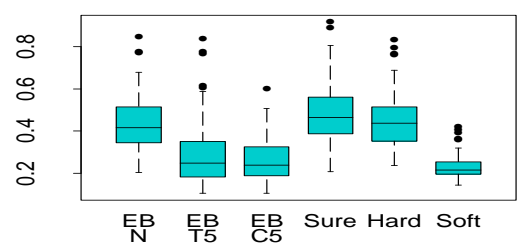

Figure 5: MSE comparisons for data simulated with $t_{5}$ errors in the wavelet domain.

be worse under heavy-tailed error distributions (recall that the signal-to-noise ratios were the same across simulations), and the number of "MSE outliers" increases dramatically. Of the three EB estimators, the performance of EB N worsened the most, indicating a stronger sensitivity to extreme errors. Compared to all the estimators, EB T5 and EB C5 performed well, offering substantial gains in efficiency for all functions; the one exception is SOFT in heavisine. The average performance of EB T5 and EB C5 is similar, although computations for EB T5 can be carried out in a fraction of the time for EB C5.

We also compared the results to the Robust-Smoother-Cleaner (RSC) transform developed by Bruce et al. (1994), which is a fast wavelet decomposition that prevents outliers and outlier patches from leaking into the wavelet coefficients at coarse levels. MSE performance of RSC in blocks, bumps, and doppler is much poorer than any of the other methods with average MSEs of 5.37, 47.13, 8.7, respectively (boxplots not included because of scale). For heavisine, the average MSE is 0.25 , which is comparable to EB C5. Visual inspections of reconstructions suggested that while RSC effectively removed outliers it often mistook signals for outliers resulting in over-smoothing, particularly in bumps.

\subsection{Student $t$ Errors in the Data Domain}

In our final set of simulations, we assessed the performance of EB N, EB T5, and EB C5 under the more realistic assumption of independent $t$ errors in the data domain. To do this we repeatedly added $n=1024$ independent $t_{5}$ errors directly to each of the four test functions, rather than to the wavelet coefficients as in the previous section, but still maintaining $\mathrm{SNR}=7$. 
blocks

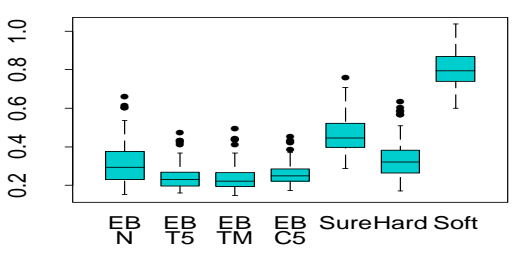

doppler

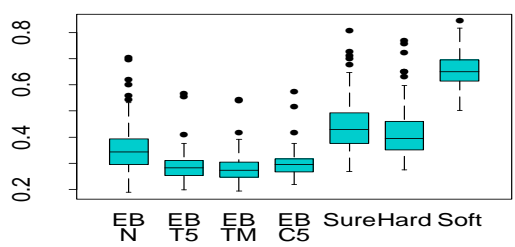

bumps

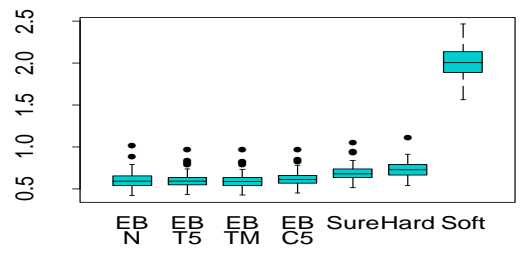

heavisine

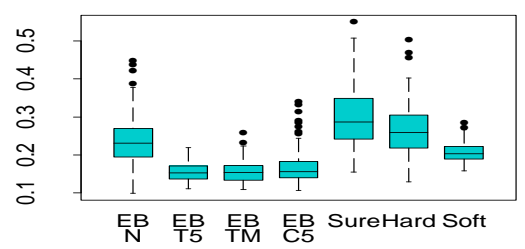

Figure 6: MSE comparisons for data simulated with $t_{5}$ errors in the data domain.

Here, the wavelet transformation induces dependent, though uncorrelated, errors in the wavelet domain that are linear combinations of $t$ random variables. Thus, the wavelet domain errors are more normally distributed, especially at the coarser levels, where the linear combinations typically involve more terms. Although a hierarchical Bayesian analysis under the exact wavelet domain error distribution is computationally impractical, one might consider a modifications of the EB T estimator to allow for increasing df at the coarser levels to account for the tendency towards normality. To explore this approach, we considered an additional estimator, labeled EB TM. The degrees of freedom for EB TM (M is for Matched) were matched to the error process by applying the wavelet transform to independent $t_{5}$ errors. For each level, we selected the df that maximized the marginal distribution of the data, and then repeated this procedure 100 times. The average degrees of freedom for the seven levels were $(5,6,6,7,7,7,8)$ (after rounding) for the "haar", "d6" and "s16" wavelet transforms, suggesting that despite the averaging due to the DWT, heavy tails can still be an issue at the coarsest levels.

Figure 6 compares the MSEs of the four EB estimators EB N, EB T5, EB TM and EB C5 with those for the SURE, HARD and SOFT estimators in this setting. Comparison with Figures 3 and 5 shows that the MSEs here are somewhat smaller here than under $t$ errors, but still much larger than under normal errors. Because of the wavelet transform, extreme values in the time domain have a smaller effect in the wavelet domain, but apparently, the tendency towards normality in the wavelet domain is not especially pronounced. All three EB estimators based on heavy tailed error distributions exhibit substantial gains in 
efficiency over the standard methods, with EB TM performing only marginally better than EB T5 or EB C5. With the exception of the SOFT estimator for the heavisine function, EB $\mathrm{N}$ offers improvement over SURE, HARD and SOFT. As in the previous section, RSC performed poorly, obtaining larger MSEs than all other methods for blocks, bumps, and doppler (average MSEs are 5.36, 47.468 .73 respectively), with the exception of heavisine, where it was comparable to EB N, but still not as good as EB T5, EB TM, or EB C5.

\section{GLINT Example}

To observe their performance on a real data set, we applied our EB estimators to the GLINT data of Bruce and Gao (1994). These data are radar reflections or glints measured in degrees for a rotating airplane model, and are subject to errors that can be as large as 150 degrees in absolute value. The true signal is supposed to be a low frequency oscillation about zero with possible level shifts, but the glint spikes are highly non-Gaussian (Bruce and Gao 1994).

We used an estimate of $\sigma=29$ so that 3 standard errors contain almost all of the glint spikes in the original signal, based on information from Bruce and Gao (1994). In Figure 7, we compare the EB estimates under the Normal-Normal and Bivariate $t$ models to hard thresholding and the Robust-Smoother-Cleaner wavelet transform of Bruce et al. (1994). In this example, shrinkage via hard thresholding has not removed the glint spikes and Bruce and Gao (1994) view the resulting estimate of the signal as quite poor. The robustsmoother-cleaner wavelet transform was designed to remove outlier patches from the wavelet decomposition, and results in a much improved, smoother reconstruction than HARD thresholding (Bruce and Gao 1994). While the EB normal estimate may still retain a spurious glint spike around $t=400$, the EB shrinkage estimates under the Bivariate $t$ model with 3 degrees of freedom have effectively removed the outliers/nonGaussian glint spikes in the original signal. Unlike the RSC wavelet transform, the EB approach is completely model based and can incorporate prior information about the process.

\section{Discussion}

In this article, we have proposed robust empirical Bayes methods for wavelet estimation. Embedding the wavelet setup in a hierarchical normal scale mixture model, we obtain maximum likelihood estimates of the

unknown hyperparameters for each wavelet level, as well as an estimate of $\sigma^{2}$ using data from all levels. We then obtained shrinkage and threshold estimators based on posterior means under the estimated prior distributions. When applied to a variety of simulated examples, these shrinkage estimators performed better 


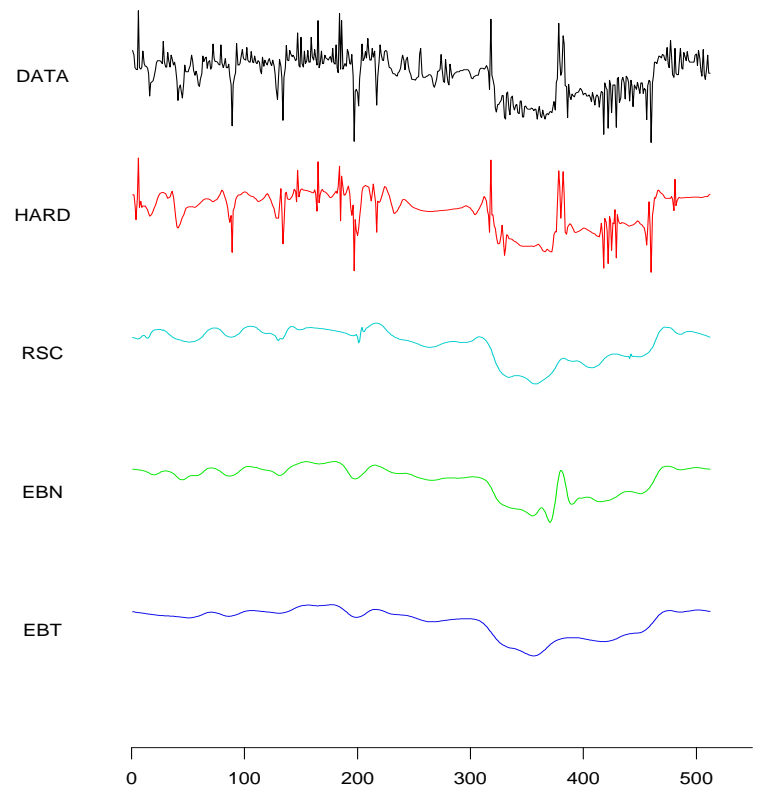

Figure 7: Shrinkage estimates for GLINT data. From top to bottom: original Glint data, hard thresholding (HARD), the robust smoother-cleaner transform (RSC), Normal EB shrinkage (EBN) and Student $t$ EB shrinkage (EBT).

than current methods including fixed parameter Bayes estimators. The EB N model provides an excellent adaptive default prior for most cases, while the EB T and EB TM approaches provide a balance between robustness when heavy tailed errors are a concern and computational ease over independent scale models in either the wavelet or original domain.

An explanation for the improved performance of the EB estimators is that they allow for wide variation of hyperparameter values across different wavelet levels, yielding flexible shrinkage patterns. The Bayesian hierarchical model can also incorporate prior uncertainty about the degrees of freedom, which leads to a posterior distribution for the degrees of freedom at each level. This avenue of research may lead to additional improvements over the current EB T or EB TM estimators in the case of heavy tailed errors in the data domain.

It would be straightforward to use our EB prior distributions and resulting posterior distributions to estimate the posterior median, as in Abramovich et al. 1998, for robust $L_{1}$ EB estimators. Analogous to Johnstone and Silverman (1997), the EB methods can be adapted to the case of correlated noise, by replacing the MLE of $\sigma$ with a level dependent MLE, $\hat{\sigma}_{j}$. Because sample sizes at coarser levels are small, 
there may be substantial uncertainty in the EB estimators of $c_{j}, w_{j}$ and $\sigma_{j}$. In cases with small signal-tonoise ratios, improved estimation can be achieved using Gibbs sampling (Clyde et al. 1998) combined with Rao-Blackwellized estimators. If the computational issues for this approach could be simplified, this would be a promising competitor to our methods, and would provide improved estimates over a naive EB approach that ignores uncertainty in the hyperparameter estimates.

\section{References}

Abramovich, F., Sapatinas, T., and Silverman, B.W. (1998). Wavelet thresholding via a Bayesian approach. Journal of the Royal Statistics Society, Series B, 60, 725-749.

Andrews, D. F. and Mallows, C. L. (1974). Scale mixtures of normal distributions. Journal of the Royal Statistical Society, Series B, 36, 99-102.

Bruce, A., Donoho, D, Gao, H-Y, and Martin, D. (1994). Denoising and robust nonlinear wavelet analysis. In SPIE Proceedings, Wavelet Applications, Volume 2242, Orlando, FL.

Bruce, A. and Gao, H-Y. (1994). S+Wavelets, Users Manual. Seattle: StatSci.

Chipman, H., Kolaczyk, E., and McCulloch, R. (1997). Adaptive Bayesian wavelet shrinkage. Journal of the American Statistical Association, 92, 1413-1421.

Clyde, M., Parmigiani, G., Vidakovic, B. (1998). Multiple shrinkage and subset selection in wavelets. Biometrika, 85, 391-402.

Dempster, A.P. Laird, N.M. and Rubin, D.B. (1977). Maximum likelihood estimation from incomplete data via the EM algorithm (with discussion). Journal of the Royal Statistical Society, Series B, 39, 1-38.

Donoho, D.L., and Johnstone, I.M., (1994). Ideal spatial adaptation by wavelet shrinkage. Biometrika, 81, $425-256$.

Donoho, D. and Johnstone, I. (1995). Adapting to unknown smoothness via wavelet shrinkage. Journal of the American Statistical Association, 90, 1200-1224.

Donoho, D., Johnstone, I., Kerkyacharian, G., and Picard, D. (1995). Wavelet shrinkage: Asymptopia? Journal of the Royal Statistical Society, series B, 57, 301-369. 
Fan, T-H, and Berger, J.O. (1990). Exact convolutions of $t$ distributions with applications to Bayesian inference for a normal mean with $t$ prior distributions. Journal of Statist. Comput. Simul., 36, $209-228$.

George, E.I. (1986). Minimax multiple shrinkage estimation. Annals of Statistics, 14, 188-205.

George, E.I. and Foster, D.P. (1997). Calibration and empirical Bayes variable selection. Tech Report, University of Texas, at Austin.

Johnstone, I.M. and Silverman, B.W. (1997). Wavelet threshold estimators for data with correlated noise. Journal of the Royal Statistical Society, Series B, 59, 319-351.

Johnstone, I.M. and Silverman, B.W. (1998). Empirical Bayes approaches to mixture problems and wavelet regression. Tech Report. University of Bristol.

O'Hagan, A. (1979). On outlier rejection phenomena in Bayes inference. Journal of the Royal Statistical Society, Series B, 41, 358-367.

O’Hagan, A. (1988). Modelling with heavy tails. Bayesian Statistics 3, eds. J.M. Bernardo, M.H. DeGroot, D.V. Lindley, and A.F.M. Smith, Oxford, U.K. Clarendon Press, pp. 345-359.

Thisted, R. A. (1988). Elements of Statistical Computing. Chapman Hall.

West, M. (1984). Outlier models and prior distributions in Bayesian linear regression. Journal of the Royal Statistical Society, Series B, 46, 431-439.

West, M. (1987). On scale mixtures of normal distributions. Biometrika, 74, 646-648. 